\title{
Research Article \\ Effect of Awareness Programs on the Epidemic Outbreaks with Time Delay
}

\author{
Lixia Zuo and Maoxing Liu \\ Department of Mathematics, North University of China, Taiyuan, Shanxi 030051, China \\ Correspondence should be addressed to Maoxing Liu; liumxsx@gmail.com
}

Received 25 February 2014; Revised 12 June 2014; Accepted 13 June 2014; Published 13 July 2014

Academic Editor: Zhichun Yang

Copyright ( 2014 L. Zuo and M. Liu. This is an open access article distributed under the Creative Commons Attribution License, which permits unrestricted use, distribution, and reproduction in any medium, provided the original work is properly cited.

\begin{abstract}
An epidemic model with time delay has been proposed and analyzed. In this model the effect of awareness programs driven by media on the prevalence of an infectious disease is studied. It is assumed that pathogens are transmitted via direct contact between the susceptible and the infective populations and further assumed that the growth rate of cumulative density of awareness programs increases at a rate proportional to the infective population. The model is analyzed by using stability theory of differential equations and numerical simulations. Both equilibria have been proved to be globally asymptotically stable. The results we obtained and numerical simulations suggest the increasing of the dissemination rate and implementation rate can reduce the proportion of the infective population.
\end{abstract}

\section{Introduction}

Infectious diseases that cause mortality, disability, and social and economic disruption are a major threat to mankind, which are responsible for a quarter of all deaths annually in the world [1-3]. Once an infectious disease appears and spreads in a region, the Center of Disease Control and Prevention will do its best to stop the propagation of the disease [4]. One of the measures is to tell people the appropriate preventive knowledge of diseases as soon as possible through media and education which make people take precautions to reduce their chances of being infected [5]. As the awareness disseminates, people will change their behaviors to alter their susceptibility. There is much evidence that media coverage can play an important role in the spread and control of infectious diseases [6-11]. In [8] Cui and others established a framework of transmission coefficient with media coverage which is a decreasing function of the number of the infective individuals and they observed a classic threshold-type behavior, with the disease becoming extinct when $R_{0}<1$ and going to a globally asymptotically stable equilibrium when $R_{0}>1$, and they concluded that media coverage is critical in disease eradication.

The models studying the spread of infectious diseases are very useful in evaluating strategies to control the diseases in populations. Recently some authors studied the impacts of media coverage and education on the spread of infectious diseases in a given region [12-14]. In [12] Misra et al. proposed a nonlinear mathematical model for the effects of awareness programs on the spread of infectious diseases and assumed the growth rate of awareness programs impacting the population is proportional to the number of infective individuals. The model analysis showed that the spread of an infectious disease can be controlled by using awareness programs but the disease remains endemic due to immigration. Yorke and London [15] proposed an SIS type compartmental model for sexually transmitted infections with the assumption that the whole population is aware of risk but only a certain proportion choose to respond by limiting their contacts with the infective population. As a result the spread of infection is controlled, leading to a reduction of the number of individuals becoming infected. A nonlinear mathematical model with delay to capture the dynamics of effect of awareness programs on the prevalence of any epidemic is proposed and analyzed [16], which assumed it increases at a rate proportional to the number of the deaths of infective individuals.

Some scholars focus on the contact rate and most of them assume that media campaigning will aid in modifying the contact rate between susceptible and infective individuals 
[17-27]. To prevent the unboundedness of the contact rate, Capasso [17] used a saturated incidence rate of the form $\beta S I /(1+\beta \delta I)$; Liu and coworkers $[23,24]$ used a nonlinear incidence rate given by $k I^{l} S /\left(1+\alpha I^{h}\right)$ to incorporate the effect of behavioral changes. In [25], the authors study how media coverage influences the dynamics of infectious disease by using SIRS model with the contact rate $\left(\beta_{1}-\beta_{2} I /(m+\right.$ $I)$ ), where $\beta_{1}, \beta_{2}$, and $m$ are positive constants. Tchuenche and Bauch used an exponentially decreasing function $e^{-M(t)}$ to reveal the force of media [27]. Cui et al. used a similar function as [13] and developed an SIR model using incidence rate $\mu e^{(-m I)} S I$ to investigate the impact of media coverage on the transmission [28]. Stability analysis of the model has shown that Hopf bifurcation can occur which causes oscillatory phenomena when $m$ is sufficiently small. Numerical simulations suggested that the media impact was stronger when the basic reproduction number $R_{0}>1$. Liu et al. [29] described the impact of media coverage using the transmission coefficient $\beta e^{\left(-\alpha_{1} E-\alpha_{2} I-\alpha_{3} H\right)}$, where $H$ is the number of hospitalized individuals. And this impact leads to the change of avoidance and contact patterns at both individual and community levels. Liu and Xiao [30] introduce a segmented function to describe the saturated media impact $e^{-m I_{c}}$ when formulating an epidemic model. A Filippov epidemic model with media coverage is proposed to describe the real characteristics of media impact in the spread of infectious diseases by incorporating a piecewise continuous transmission rate $\beta e^{(-\alpha \epsilon I)} S I$ in [31]. Mathematical and bifurcation analysis with regard to the local, global stability of equilibria and local sliding bifurcations are performed. Bhunu et al. [32] and Tchuenche and Bauch[27] focused on the different types of population in their work.

In order to better describe population mixed conditions, some authors study infectious diseases models on the different networks $[33,34]$. Funk et al. have overlaid the model of information spread of a contagious disease on two, not necessarily identical networks, with more informed individuals acting to reduce their susceptibility [35]. Liu et al. took into the random perturbation [36]. In [37], the authors extended the classical SIRS epidemic model from a deterministic framework to a stochastic differential equation, and then they gave the conditions of existence of unique positive solution and the stochastic extinction and discussed the exponential $p$-stability and global stability.

Most of the articles, such as [12], assume that, due to awareness programs, driven by media, some susceptible individuals will avoid their contacts with the infectious individuals resulting in formation of a new class in the population and this newly formed aware class may contract infection only if they lose awareness. But we regard it is unreasonable. In fact sometimes even if persons are conscious of diseases, they will also be infected. Therefore we propose a mathematical model for predicting the future course of any epidemic by considering this newly formed aware class into the model.

The rest of this paper is organized as follows. In the next section, to capture the dynamics of effect of cumulative density of awareness programs on the prevalence of any epidemic a mathematical model is proposed and analyzed. Then we analyze the local and global stability of the disease-free and the unique endemic equilibrium in Section 3. Furthermore, in Section 4 we perform some numerical examples to validate the analytical findings in Section 3 and introduce the importance of the dissemination rate and implementation rate in disease control. In Section 5 we discuss the above content.

\section{Mathematical Model and Equilibrium Analysis}

In this paper due to the awareness programs about the disease, susceptible individuals are rarely in contact with the infective ones and form a different class, namely, aware susceptible class; thus the total population is divided into three classes, the susceptible population, the aware population, and the infective population, and the proportions of them in the total population are $X(t), Y(t)$, and $X_{m}(t)$. Assuming that at time $t$ the cumulative density of awareness programs driven by media in that region is $M(t)$, which increases at a rate proportional $\mu$ to the infective population and consumes due to causes like inefficiency and psychological barriers at $\mu_{0}$, thus

$$
M^{\prime}(t)=\mu Y(t)-\mu_{0} M(t) .
$$

In fact people cannot take measures to protect themselves in time after the media reports the disease, so we introduce a time delay $\tau$ that represents the interval between the report time and the time of taking measures. We assume that a proportion of infected individuals recover through treatment and, after recovery, a fraction $q$ of recovered people will become aware and join the aware susceptible class whereas the remaining fraction $p(p+q=1)$ will become unaware susceptible. Keeping the above facts in mind, the dynamics of a model are governed by the following systems of nonlinear ordinary differential equations:

$$
\begin{aligned}
X^{\prime}(t)= & b-\beta X(t) Y(t)+\lambda_{0} X_{m}(t) \\
& -\lambda X(t) M(t-\tau)-d X(t)+p \nu Y(t), \\
X_{m}^{\prime}(t)= & \lambda X(t) M(t-\tau)-\left(\lambda_{0}+d\right) X_{m}(t) \\
& -\alpha X_{m}(t) Y(t)+q \nu Y(t) \\
Y^{\prime}(t)= & \beta X(t) Y(t)-(\nu+d) Y(t) \\
& +\alpha X_{m}(t) Y(t) \\
M^{\prime}(t)= & \mu Y(t)-\mu_{0} M(t) .
\end{aligned}
$$

Here $X(0)>0, Y(0) \geq 0$, and $X_{m}(0) \geq 0$.

Assume diseases spread due to the direct contact between susceptible and infective individuals only. In the above model, the rate of immigration of susceptible population is $b . \alpha$ is the contact rate of aware susceptible with infective population and $\beta(\alpha<\beta)$ is the contact rate of unaware ones. The constant $\lambda$ represents the dissemination rate of awareness among unaware susceptible due to which they form a different class; then $\lambda_{0}$ denotes the rate of transfer of aware susceptible 
to unaware class. The constants $v, d$ represent the recovery rate and the natural death rate, respectively. All the above constants are assumed to be positive. Using the fact that $X(t)+$ $X_{m}(t)+Y(t)=1$, the system (2) becomes as follows:

$$
\begin{aligned}
X_{m}^{\prime}(t)= & \lambda\left(1-X_{m}(t)-Y(t)\right) M(t-\tau) \\
& -\left(\lambda_{0}+d\right) X_{m}(t)-\alpha X_{m}(t) Y(t)+q \nu Y(t), \\
Y^{\prime}(t)= & \beta\left(1-X_{m}(t)-Y(t)\right) Y(t) \\
& -(\nu+d) Y(t)+\alpha X_{m}(t) Y(t), \\
M^{\prime}(t)= & \mu Y(t)-\mu_{0} M(t) .
\end{aligned}
$$

Now it is sufficient to study system (3) in detail rather than system (2).

For the analysis of system (3), we need the region of attraction which is given by the set: $\Omega=\left\{\left(X_{m}, Y, M\right) \in \mathfrak{R}_{+}^{3}\right.$ : $\left.0 \leq X_{m}, Y \leq 1,0 \leq M<\mu / \mu_{0}\right\}$, which attracts all solutions initiating in the interior of the positive orthant.

Define the basic reproduction number $R_{0}=\beta /(\nu+$ $d)$. There are two equilibria, the disease-free equilibrium $E_{0}(0,0,0)$ and the endemic equilibrium $E_{*}\left(X_{m}^{*}, Y^{*}, M^{*}\right)$; the existence of $E_{0}$ is trivial; then we prove the existence of $E_{*}$ in detail. In $E_{*}\left(X_{m}^{*}, Y^{*}, M^{*}\right)$ the values of $X_{m}^{*}, M^{*}$ are obtained by solving the following algebraic equations (for $Y \neq 0$ ):

$$
\begin{gathered}
\lambda\left(1-X_{m}-Y\right) M-\left(\lambda_{0}+d\right) X_{m}-\alpha X_{m} Y+q \nu Y=0 \\
\beta\left(1-X_{m}-Y\right) Y-(\nu+d) Y+\alpha X_{m} Y=0 \\
\mu Y-\mu_{0} M=0
\end{gathered}
$$

Using (5) and (6), we get

$$
\begin{gathered}
X_{m}^{*}=\frac{\beta\left(1-Y^{*}\right)-(\nu+d)}{\beta-\alpha}, \\
M^{*}=\frac{\mu}{\mu_{0}} Y^{*} .
\end{gathered}
$$

Further, using (7) and (8) in (4), we obtain a quadratic equation in $Y^{*}$ as

$$
P_{1} Y^{* 2}+P_{2} Y^{*}+P_{3}=0
$$

where

$$
\begin{aligned}
P_{1}= & \alpha\left(\lambda \mu+\mu_{0} \beta\right), \\
P_{2}= & (\nu+d-\beta)\left(\lambda \mu+\alpha \mu_{0}\right) \\
& +q \nu \mu_{0}(\beta-\alpha)+\mu_{0} \beta(\beta+d), \\
P_{3}= & \mu_{0}\left(\lambda_{0}+d\right)(\nu+d-\beta) .
\end{aligned}
$$

Solving (9) we get

$$
Y^{*}=\frac{-P_{2} \pm \sqrt{P_{2}^{2}-4 P_{1} P_{3}}}{2 P_{1}} .
$$

We obtain $P_{1}>0$ and $P_{3}<0$ when $R_{0}>1$ and get $Y^{*}=$ $\left(-P_{2}+\sqrt{P_{2}^{2}-4 P_{1} P_{3}}\right) / 2 P_{1}$ for $Y^{*}>0$.

Remark. From the expression of $Y^{*}$, it is easy to note that $\left(d Y^{*} / d \lambda\right)<0$ and $\left(d Y^{*} / d \mu\right)<0$, which shows that the equilibrium number of infective individuals decreases as the rate of dissemination and the implementation rate of awareness programs increase.

\section{Stability Analysis}

In this section we present the local and global stability of $E_{0}$ and $E_{*}$.

\subsection{The Stability of the Disease-Free Equilibrium}

Theorem 1. When $\tau \geq 0$, the disease-free equilibrium $E_{0}(0,0,0)$ is locally asymptotically stable if $R_{0}<1$ and becomes unstable if $R_{0}>1$.

Proof. The Jacobian matrix corresponding to system (4) is given as follows:

$$
J=\left[\begin{array}{ccc}
-\lambda M-\alpha Y-\lambda_{0}-d & -\lambda M-\alpha X_{m}+q \nu & \lambda\left(1-Y-X_{m}\right) e^{-\eta \tau} \\
(\alpha-\beta) Y & (\alpha-\beta) X_{m}-2 \beta Y+(\beta-\nu-d) & 0 \\
0 & \mu & -\mu_{0}
\end{array}\right]
$$

where $\eta$ is the eigenvalue. Then the characteristic equation is

$$
\left[\eta+\left(\lambda_{0}+d\right)\right][\eta+(\nu+d-\beta)]\left(\eta+\mu_{0}\right)=0
$$

We get $\eta_{1}=-\left(\lambda_{0}+d\right)<0, \eta_{2}=\beta-(\nu+d)$, and $\eta_{3}=-\mu_{0}<0$. So $\eta_{2}<0$ when $R_{0}<1 ; \eta_{2}>0$ when $R_{0}>1$.

Theorem 2. When $\tau \geq 0$, the disease-free equilibrium $E_{0}$ is globally asymptotically stable in $\Omega$ if $R_{0}<1$.
Proof. To establish the global stability of the disease-free equilibrium $E_{0}$, we use Lyapunov's method and consider the following positive definite function without $\tau$ :

$$
V=\frac{1}{2} Y^{2}
$$

Now differentiating $V$ with respect to $t$, we get

$$
\frac{d V}{d t}=[\beta-(\nu+d)] Y^{2}-\beta Y^{3}-(\beta-\alpha) X_{m} Y^{2} \text {. }
$$


When $R_{0}<1, d V / d t \leq 0$. The largest compact invariant set in $\left\{\left(X_{m}, Y, M\right) \in \Omega: V^{\prime}=0\right\}$ when $R_{0}<1$ is the singleton $\left\{E_{0}\right\}$. Then LaSalle's invariance principle implies that $E_{0}$ is globally asymptotically stable in $\Omega$.

3.2. The Stability of the Endemic Equilibrium. Linearizing system (4) about $E_{*}$, let $x=X_{m}-X_{m}^{*}, y=Y-Y^{*}$, and $m=M-M^{*}$ and get

$$
\frac{d u}{d t}=M_{1} u(t)+M_{2} u(t-\tau)
$$

where

$$
\begin{gathered}
u(t)=[x(t), y(t), m(t)]^{T}, \\
M_{1}=\left[\begin{array}{ccc}
-\left(\lambda M^{*}+\alpha Y^{*}+\lambda_{0}+d\right) & q v-\lambda M^{*}-\alpha X_{m}^{*} & 0 \\
(\alpha-\beta) Y^{*} & -\beta Y^{*} & 0 \\
0 & \mu & -\mu_{0}
\end{array}\right], \\
M_{2}=\left[\begin{array}{ccc}
0 & 0 & \lambda\left(1-Y^{*}-X_{m}^{*}\right) \\
0 & 0 & 0 \\
0 & 0 & 0
\end{array}\right] .
\end{gathered}
$$

We have the disease-free equilibrium $E_{0}^{\prime}(0,0,0)$ and the endemic equilibrium $E_{*}^{\prime}\left(x^{*}, y^{*}, m^{*}\right)$, where the stability of $E_{*}$ about system (3) is corresponding with $E_{0}^{\prime}$. The characteristic equation of the above system at $E_{0}^{\prime}$ is

$$
\eta^{3}+Q_{1} \eta^{2}+Q_{2} \eta+Q_{3}=Q_{4} e^{-\eta \tau}
$$

where $\eta$ is the eigenvalue and

$$
\begin{aligned}
Q_{1} & =A+\beta Y^{*}+\mu_{0}, \\
Q_{2} & =\beta Y^{*} A+\left(A+\beta Y^{*}\right) \mu_{0}+(\alpha-\beta) Y^{*} B, \\
Q_{3} & =\beta Y^{*} \mu_{0} A+(\alpha-\beta) \mu_{0} Y^{*} B, \\
Q_{4} & =(\beta-\alpha) \mu Y^{*} C, \\
A & =\lambda M^{*}+\alpha Y^{*}+\lambda_{0}+d, \\
B & =\lambda M^{*}+\alpha X_{m}^{*}-q \nu, \\
C & =\lambda\left(Y^{*}+X_{m}^{*}-1\right) .
\end{aligned}
$$

The stability of the endemic equilibrium $E_{*}$ of system (3) is stated in the following theorems.

Theorem 3. When $\tau \geq 0$, the endemic equilibrium $E_{*}$ is locally asymptotically stable if $R_{0}>1$.

Proof. When $\tau=0$, the characteristic equation is of the form

$$
\eta^{3}+Q_{1} \eta^{2}+Q_{2} \eta+\left(Q_{3}-Q_{4}\right)=0
$$

It is easy to see that

$$
\begin{aligned}
& Q_{1}=A+\beta Y^{*}+\mu_{0} \\
& =\lambda M^{*}+\alpha Y^{*}+\lambda_{0}+d+\beta Y^{*}+\mu_{0}>0, \\
& Q_{2}=\beta Y^{*} A+\left(A+\beta Y^{*}\right) \mu_{0}+(\alpha-\beta) Y^{*} B \\
& =\beta Y^{*} \lambda M^{*}+\beta Y^{*} \alpha Y^{*}+\beta Y^{*}\left(\lambda_{0}+d\right) \\
& +\beta Y^{*} \mu_{0}+\lambda M^{*}+\alpha Y^{*}+\lambda_{0}+d \\
& +(\alpha-\beta) Y^{*} \lambda M^{*}+(\alpha-\beta) Y^{*} \alpha X_{m}^{*} \\
& +(\beta-\alpha) Y^{*} v q \\
& =\alpha \lambda Y^{*} M^{*}+\alpha^{2} Y^{*} X_{m}^{*}+\alpha Y^{*}\left(1-X_{m}^{*}\right) \\
& +(\beta-\alpha) v q Y^{*}+\alpha \beta Y^{* 2}+\beta Y^{*}\left(\lambda_{0}+d\right) \\
& +\beta \mu_{0} Y^{*}+\lambda M^{*}+\lambda_{0}+d>0 \text {. } \\
& Q_{3}-Q_{4} \\
& =\beta Y^{*} \mu_{0} A+(\alpha-\beta) \mu_{0} Y^{*} B \\
& +(\alpha-\beta) \mu Y^{*} C \\
& =\beta Y^{*} \mu_{0} \lambda M^{*}+\beta Y^{*} \mu_{0} \alpha Y^{*} \\
& +\beta Y^{*} \mu_{0}\left(\lambda_{0}+d\right)+\alpha \mu_{0} Y^{*} \lambda M^{*} \\
& +\alpha \mu_{0} Y^{*} \alpha X_{m}^{*}-\alpha \mu_{0} Y^{*} v q-\beta \mu_{0} Y^{*} \lambda M^{*} \\
& -\beta \mu_{0} Y^{*} \alpha X_{m}^{*}+\beta \mu_{0} Y^{*} v q-(\beta-\alpha) \mu Y^{*} C \\
& =(\beta-\alpha) \mu_{0} v q Y^{* 2}+\mu_{0} \alpha \beta X_{m}^{*} Y^{*} x^{*}\left(y^{*}\right)^{-1} \\
& +\beta \mu_{0} \alpha Y^{* 2}+\beta \mu_{0}\left(\lambda_{0}+d\right) Y^{*} \\
& +\alpha \lambda \mu_{0} Y^{*} M^{*}+(\beta-\alpha) \lambda \mu Y^{*}\left(1-Y^{*}-X_{m}^{*}\right)>0, \\
& Q_{1} Q_{2}-\left(Q_{3}-Q_{4}\right) \\
& =\left(A+\beta Y^{*}+\mu_{0}\right)\left[\beta Y^{*} A+\left(A+\beta Y^{*}\right) \mu_{0}+(\alpha-\beta) Y^{*} B\right] \\
& -\left[\beta Y^{*} \mu_{0} A+(\alpha-\beta) \mu_{0} Y^{*} B-(\beta-\alpha) \mu Y^{*} C\right] \\
& =\left(A+\beta Y^{*}+\mu_{0}\right) \beta Y^{*} A+A^{2} \mu_{0} \\
& +\left(\beta Y^{*}+\mu_{0}\right)\left(A+\beta Y^{*}\right) \mu_{0} \\
& +\left(A+\beta Y^{*}\right)(\alpha-\beta) Y^{*} B+(\alpha-\beta) Y^{*} \mu C \\
& =(\beta-\alpha) \mu \lambda Y^{*}\left(Y^{*}+X_{m}^{*}\right)+\alpha \lambda \mu Y^{*} \\
& +\left(A+\beta Y^{*}\right)\left[A \beta Y^{*}+(\alpha-\beta) Y^{*} B\right] \\
& +(\beta-\alpha) \mu \lambda Y^{*} x^{*}\left(y^{*}\right)^{-1}+\mu_{0} \beta Y^{*} A \\
& +A^{2} \mu_{0}+\left(\beta Y^{*}+\mu_{0}\right)\left(A+\beta Y^{*}\right) \mu_{0} .
\end{aligned}
$$


From $Q_{3}-Q_{4}>0$, we can get $A \beta Y^{*}+(\alpha-\beta) Y^{*} B>0$; thus $Q_{1} Q_{2}-\left(Q_{3}-Q_{4}\right)>0$. According to Hurwitz criterion, we can know all the $\eta$ 's have negative real parts; then $E_{0}^{\prime}$ is locally asymptotically stable.

When $\tau>0$, notice that (18) does not have nonnegative real roots. If it has roots with nonnegative real parts they must be complex and should have been obtained from a pair of complex conjugate roots which cross the imaginary axis. Consequently, (18) must have a pair of purely imaginary solutions for some $\tau>0$. Assume that $\eta=i \omega(\omega>0)$ is a root of (18) without loss of generality. That is the case if and only if $\omega$ satisfies the equation

$$
-\omega^{3} i-Q_{1} \omega^{2}+Q_{2} \omega i+Q_{3}=Q_{4}(\cos \omega \tau-i \sin \omega \tau) .
$$

Separating the real and imaginary parts, we have the following system, satisfied by $\omega$ :

$$
\begin{aligned}
& Q_{3}-Q_{1} \omega^{2}=Q_{4} \cos \omega \tau, \\
& \omega^{3}-Q_{2} \omega=Q_{4} \sin \omega \tau .
\end{aligned}
$$

To eliminate the trigonometric functions we square both sides of each equation above and we add the squared above equations to obtain the following forth order equation in $\omega$ :

$$
\omega^{6}+\left(Q_{1}^{2}-2 Q_{2}\right) \omega^{4}+\left(Q_{2}^{2}-2 Q_{1} Q_{3}\right) \omega^{2}+\left(Q_{3}^{2}-Q_{4}^{2}\right)=0
$$

To reduce this fourth order equation in $\omega$ to a quadratic equation let $\varphi=\omega^{2}$ and denote the coefficients by

$$
\varphi^{3}+R_{1} \varphi^{2}+R_{2} \varphi+R_{3}=0
$$

where

$$
\begin{aligned}
R_{1}= & Q_{1}^{2}-2 Q_{2} \\
= & \left(A+\beta Y^{*}+\mu_{0}\right)^{2} \\
& -2\left[\beta Y^{*} A+\left(A+\beta Y^{*}\right) \mu_{0}+(\alpha-\beta) Y^{*} B\right] \\
= & A^{2}+\beta^{2} Y^{* 2}+\mu_{0}^{2}+2(\beta-\alpha) Y^{*} B \\
= & A^{2}+\beta^{2} Y^{* 2}+\mu_{0}^{2}+2(\beta-\alpha) Y^{*}\left(\lambda M^{*}+\alpha X_{m}^{*}\right) \\
& +2 \nu q \beta Y^{*} y^{*}\left(x^{*}\right)^{-1}>0, \\
R_{2}= & Q_{2}^{2}-2 Q_{1} Q_{3} \\
= & {\left[\beta Y^{*} A+(\alpha-\beta) Y^{*} B\right]^{2} } \\
& +\mu_{0}^{2}\left[A^{2}+\beta^{2} Y^{* 2}+(\beta-\alpha) Y^{*} B\right]
\end{aligned}
$$

$$
\begin{aligned}
&= {\left[\beta Y^{*} A+(\alpha-\beta) Y^{*} B\right]^{2} } \\
&+\mu_{0}^{2}\left[A^{2}+\beta^{2} Y^{* 2}(\beta-\alpha) Y^{*}\left(\lambda M^{*}+\alpha X_{m}^{*}\right)\right. \\
&\left.\quad+2 v q \beta Y^{*} y^{*}\left(x^{*}\right)^{-1}\right]>0 . \\
& R_{3}= Q_{3}^{2}-Q_{4}^{2}=\left(Q_{3}+Q_{4}\right)\left(Q_{3}-Q_{4}\right), \\
& Q_{3}+Q_{4} \\
&=\beta Y^{*} \mu_{0} A+(\alpha-\beta) \mu_{0} Y^{*} B+(\beta-\alpha) \mu Y^{*} C \\
&=\beta Y^{*} \mu_{0} A+(\beta-\alpha) \mu_{0} Y^{*} v q \\
&+(\alpha-\beta) \mu_{0} Y^{*}\left(\lambda M^{*}+\alpha X_{m}^{*}\right) \\
&+(\beta-\alpha) \mu Y^{*} \lambda\left(Y^{*}+X_{m}^{*}\right)+(\alpha-\beta) \mu Y^{*} \lambda \\
&=\beta Y^{*} \mu_{0}\left(\lambda M^{*}+\alpha Y^{*}+\lambda_{0}+b\right)+(\beta-\alpha) \mu_{0} Y^{*} v q \\
&+\left(\mu_{0} \lambda M^{*}+\mu_{0} \alpha X_{m}^{*}+\mu \lambda\right) \lambda Y^{*} y^{*}\left(x^{*}\right)^{-1} \\
&+(\beta-\alpha) \mu Y^{*} \lambda\left(Y^{*}+X_{m}^{*}\right)>0 .
\end{aligned}
$$

So all the coefficients of (25) are positive numbers. Then according to Lemma 3.3.1 in [38], (25) has no positive real roots; that is, we may not get any positive value of $\omega$, which satisfy the transcendental equation (18). So all the $\eta$ 's have negative real parts for all values of the delay $\tau \geq 0$; then $E_{0}^{\prime}$ is locally asymptotically stable. Thus when $\tau \geq 0, E_{*}$ is locally asymptotically stable if $R_{0}>1$.

Theorem 4. When $\tau \geq 0$, the endemic equilibrium $E_{*}$ is globally asymptotically stable in $\Omega$ if $R_{0}>1$.

Proof. Using Lyapunov's method, we consider the following positive function:

$$
V=\frac{1}{2} y^{2}
$$

The derivative of $V$ along the system is given by

$$
\frac{d V}{d t}=(\alpha-\beta) Y^{*} x-\beta Y^{*} y \leq 0
$$

The largest compact invariant set when $V^{\prime}=0$ is the singleton $\left\{E_{0}^{\prime}\right\}$. Then LaSalle's invariance principle implies that $E_{0}^{\prime}$ is globally asymptotically stable; that is, $E_{*}$ is globally asymptotically stable in $\Omega$.

\section{Numerical Simulations and Results}

To check the feasibility of our analysis of $\tau>0$, we present some numerical computations in this section using Matlab by choosing the following set of parameter values: $\beta=0.35$, $\lambda=0.08, \lambda_{0}=0.02, \alpha=0.2, d=0.002, v=0.43, p=0.15$, $q=0.85, \mu=0.002, \mu_{0}=0.02$, and $\tau=1$ when $R_{0}<1$. Let $\beta=0.5$; it may be checked that the condition $R_{0}>1$ 


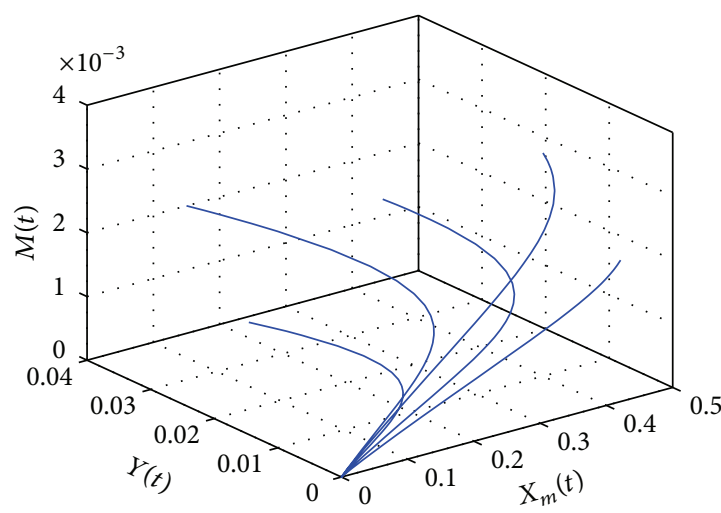

(a)

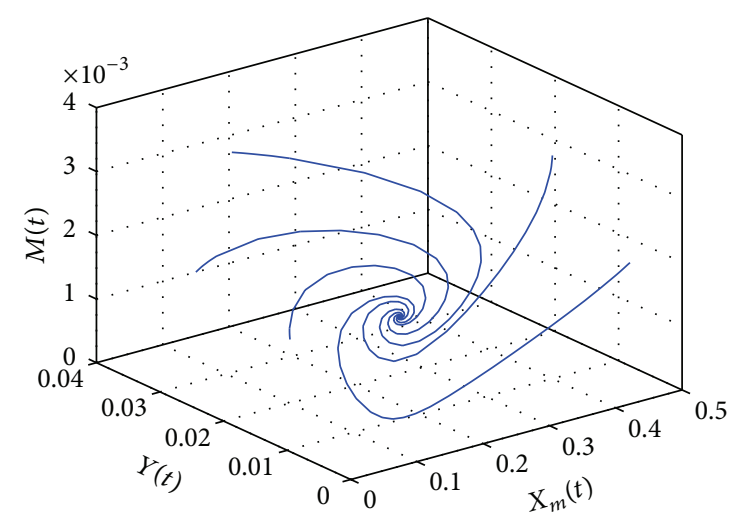

(b)

FIgURE 1: The stability of $E_{0}$ and $E_{*}$ with different initial values.

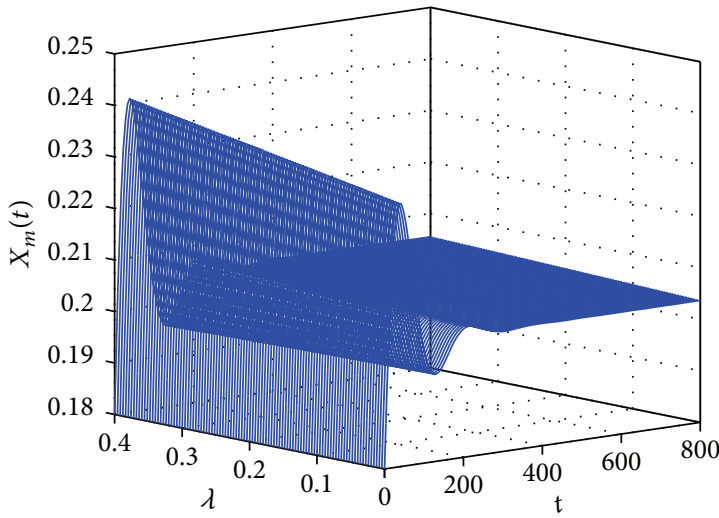

(a)

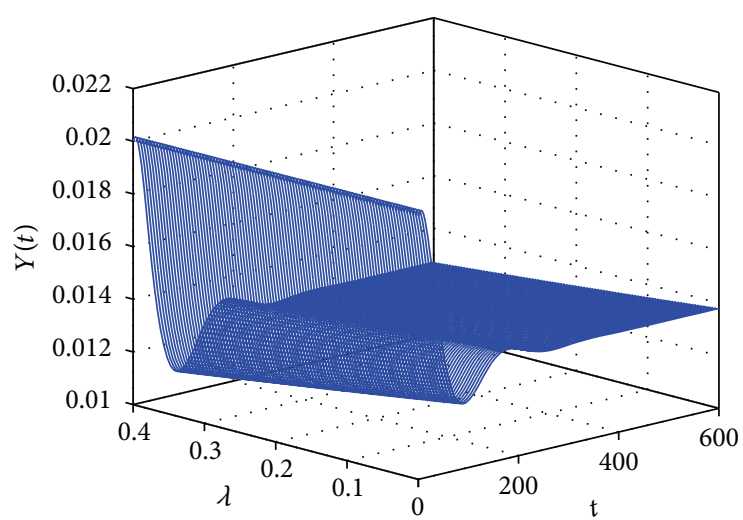

(b)

FIgure 2: The stability of $X_{m}, Y$ with variational $\lambda$.

of existence of the endemic equilibrium $E_{*}$. The equilibrium values for this data are obtained as

$$
X_{m}^{*}=0.201, \quad Y^{*}=0.014, \quad M^{*}=0.001 .
$$

The basic reproduction number $R_{0}$, for the above set of parameter values, is found to be 1.157 .

For the above parameter values, we select five sets of different initial starts; then the computer generated graphs of aware population, infective population, and cumulative density of awareness programs, respectively, have been drawn in Figure 1, which shows that all the trajectories initiating inside the region of attraction approach towards $E_{0}$ and $E_{*}$, respectively. Both of the equilibria $E_{0}$ and $E_{*}$ are locally asymptotically stable for given set of parameter values that numerical simulations support the analysis given in Section 3. In fact, they are globally asymptotically stable in $\Omega$ as we have proved.

In the following, we research the relationships of $X_{m}^{*}, Y^{*}$ and the dissemination rate $\lambda$, the implementation rate $\mu$ separately. We make $\lambda$, $\mu$ change from 0 to 0.4 and get the performances of $X_{m}(t)$ and $Y(t)$, the trajectories of which with respect to time $t$ for different $\lambda$ and $\mu$ are shown in Figures 2 and 3, respectively. And there are no awareness programs when $\lambda$ and $\mu$ are equal to zero. As shown in Figures 2 and
3, $X_{m}^{*}$ both increase and $Y^{*}$ both reduce as the increase of $\lambda$ and $\mu$, which proves the conclusions of the remark. And $\lambda, \mu$ are greater influence on $Y^{*}$ than $X_{m}^{*}$, which state awareness programs have a positive effect on prevention of diseases. In addition the reason why $X_{m}^{*}\left(Y^{*}\right)$ has a similar trend as the variations of $\lambda$ and $\mu$ is that $\lambda$ and $\mu$ have a similar influence on $X_{m}^{*}\left(Y^{*}\right)$. From the figure we obtain that $\mu$ can postpone the time of the balance of equilibrium; thus we can have more time to formulate measures to prevent diseases. There really is an effort here to make it clear that $\lambda$ and $\mu$ (awareness programs) play a key role in the prevention and control of diseases.

\section{Discussion}

The media is widely acknowledged as a key tool for influencing people's behaviors towards the disease to devise proper policies for controlling the epidemic. Awareness programs through media make people be aware about the disease and take various precautions to reduce their chances of being infected. In this paper, we propose and analyze a mathematical model to study the effect of awareness programs driven by media and the delay on the prevalence 


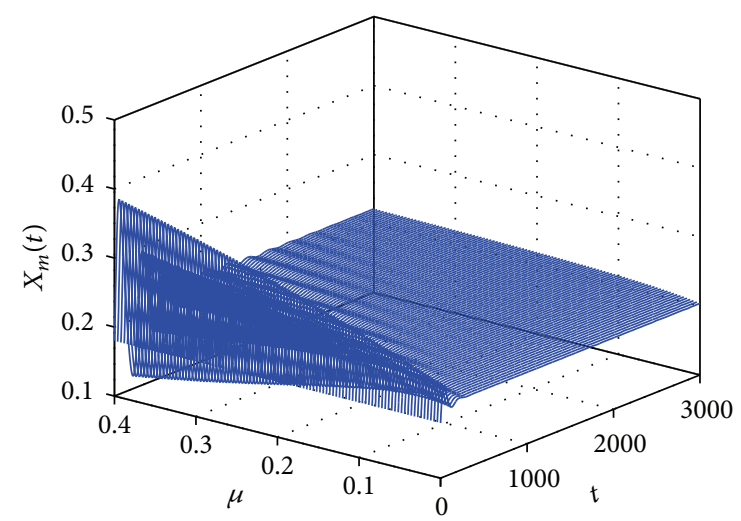

(a)

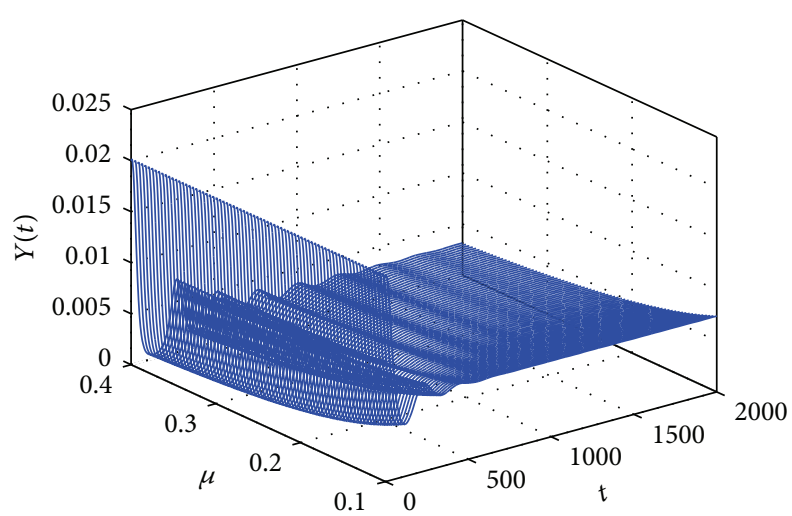

(b)

FIgURE 3: The stability of $X_{m}, Y$ with variational $\mu$.

of an infectious disease. It is assumed that pathogens are transmitted via direct contact between the susceptible and the infective populations. Assume further that cumulative density of awareness programs increases at a rate proportional to the infective population. The model exhibits two equilibria; the disease-free equilibrium has been shown to be stable for basic reproduction number $R_{0}<1$. For $R_{0}>1$, it becomes unstable, which leads to the existence of an endemic equilibrium. The endemic equilibriums are globally asymptotically stable. The delay $\tau$ has no effect on the stability of the system. The numerical simulations and results that prove the stability of equilibria suggest that if we want to reduce the proportions of the infective population and increase the aware population, we can increase the dissemination rate $\lambda$ and implementation rate $\mu$. They are conducive to controlling the spread of diseases.

\section{Conflict of Interests}

The authors declare that there is no conflict of interests regarding the publication of this paper.

\section{Acknowledgments}

This work is supported partially by the National Natural Science Foundation of China (no. 10901145) and the Natural Science Foundation of Shanxi (2012011002-1).

\section{References}

[1] World Health Organization, "Global burden of disease: 2004 update," 2008, http://www.who.int/healthinfo/global-burdendisease/2004reportupdate/en/.

[2] World Health Organization, "Report on the Global AIDS Epidemic," 2008, http://www.unaids.org/en/KnowledgeCentre/ HIVData/GlobalReport/2008/2008 Global report.asp.

[3] J. G. Breman, M. S. Alilio, and A. Mills, "Conquering the intolerable burden of malaria: what's new, what's needed: a summary," American Journal of Tropical Medicine and Hygiene, vol. 71, no. 2, pp. 1-15, 2004.
[4] R. Laxminarayan, A. J. Mills, J. G. Breman et al., "Advancement of global health: key messages from the Disease Control Priorities Project," Lancet, vol. 367, no. 9517, pp. 1193-1208, 2006.

[5] P. L. M. Vasterman and N. Ruigrok, "Pandemic alarm in the Dutch media: media coverage of the 2009 influenza A (H1N1) pandemic and the role of the expert sources," European Journal of Communication, vol. 28, no. 4, pp. 436-453, 2013.

[6] D. Gao and S. Ruan, "An SIS patch model with variable transmission coefficients," Mathematical Biosciences, vol. 232, no. 2, pp. 110-115, 2011.

[7] J. M. Tchuenche, N. Dube, C. P. Bhunu, R. J. Smith, and C. T. Bauch, "The impact of media coverage on the transmission dynamics of human influenza," BMC Public Health, vol. 11, no. 1, article S5, 2011.

[8] J. Cui, X. Tao, and H. Zhu, "An SIS infection model incorporating media coverage," The Rocky Mountain Journal of Mathematics, vol. 38, no. 5, pp. 1323-1334, 2008.

[9] Y. Li and J. Cui, "The effect of constant and pulse vaccination on SIS epidemic models incorporating media coverage," Communications in Nonlinear Science and Numerical Simulation, vol. 14, no. 5, pp. 2353-2365, 2009.

[10] Z. Mukandavire, W. Garira, and J. M. Tchuenche, "Modelling effects of public health educational campaigns on HIV/AIDS transmission dynamics," Applied Mathematical Modelling, vol. 33, no. 4, pp. 2084-2095, 2009.

[11] S. Samanta, S. Rana, A. Sharma, A. K. Misra, and J. Chattopadhyay, "Effect of awareness programs by media on the epidemic outbreaks: a mathematical model," Applied Mathematics and Computation, vol. 219, no. 12, pp. 6965-6977, 2013.

[12] A. K. Misra, A. Sharma, and J. B. Shukla, "Modeling and analysis of effects of awareness programs by media on the spread of infectious diseases," Mathematical and Computer Modelling, vol. 53, no. 5-6, pp. 1221-1228, 2011.

[13] H. Joshi, S. Lenhart, and K. Albright, "Modeling the effect of information campaigns on the HIV epidemic in Uganda," Mathematical Biosciences and Engineering, vol. 5, no. 4, pp. 757$770,2008$.

[14] F. Nyabadza, C. Chiyaka, Z. Mukandavire, and S. D. HoveMusekwa, "Analysis of an HIV/AIDS model with public-health information campaigns and individual withdrawal," Journal of Biological Systems, vol. 18, no. 2, pp. 357-375, 2010.

[15] J. A. Yorke and W. P. London, "Recurrent outbreaks of measles, chickenpox and mumps. II. Systematic differences in contact 
rates and stochastic effects," American Journal of Epidemiology, vol. 98, no. 6, pp. 469-482, 1973.

[16] I. Z. Kiss, J. Cassell, M. Recker, and P. L. Simon, "The impact of information transmission on epidemic outbreaks," Mathematical Biosciences, vol. 225, no. 1, pp. 1-10, 2010.

[17] V. Capasso, Mathematical Structure of Epidemic System, vol. 97 of Lecture Notes in Biomathematics, Springer, Berlin, Germany, 1993.

[18] H. W. Hethcote and S. A. Levin, "Periodicity in epidemiological models," in Applied Mathematical Ecology, vol. 18 of Biomathematics, pp. 193-211, 1989.

[19] S. Collinson and J. M. Heffernan, "Modelling the effects of media during an influenza epidemic," Collinson and Heffernan BMC Public Health, vol. 14, article 376, 2014.

[20] J. H. Pang and J. A. Cui, "An SIRS epidemiological model with nonlinear incidence rate incorporating media coverage," in Proceedings of the 2nd International Conference on Information and Computing Science (ICIC '09), pp. 116-119, Manchester, UK, May 2009.

[21] M. Liu, G. Röst, and G. Vas, "SIS model on homogeneous networks with threshold type delayed contact reduction," Computers \& Mathematics with Applications, vol. 66, no. 9, pp. 15341546, 2013.

[22] M. Elenbaas, H. G. Boomgaarden, A. R. T. Schuck, and C. H. de Vreese, "The impact of media coverage and motivation on performance-relevant information," Political Communication, vol. 30, no. 1, pp. 1-16, 2013.

[23] W. M. Liu, H. W. Hethcote, and S. A. Levin, "Dynamical behavior of epidemiological models with nonlinear incidence rates," Journal of Mathematical Biology, vol. 25, no. 4, pp. 359380, 1987.

[24] W. M. Liu, S. A. Levin, and Y. Iwasa, "Influence of nonlinear incidence rates upon the behavior of SIRS epidemiological models," Journal of Mathematical Biology, vol. 23, no. 2, pp. 187204, 1986.

[25] Y. Liu and J. Cui, "The impact of media coverage on the dynamics of infectious disease," International Journal of Biomathematics, vol. 1, no. 1, pp. 65-74, 2008.

[26] H. Zhao, Y. Lin, and Y. Dai, "An SIRS epidemic model incorporating media coverage with time delay," Computational and Mathematical Methods in Medicine, vol. 2014, Article ID 680743, 10 pages, 2014.

[27] J. M. Tchuenche and C. T. Bauch, "Dynamics of an infectious disease where media coverage influences transmission," ISRN Biomathematics, vol. 2012, Article ID 581274, 10 pages, 2012.

[28] J. Cui, Y. Sun, and H. Zhu, "The impact of media on the control of infectious diseases," Journal of Dynamics and Differential Equations, vol. 20, no. 1, pp. 31-53, 2008.

[29] R. S. Liu, J. H. Wu, and H. P. Zhu, "Media/psychological impact on multiple outbreaks of emerging infectious diseases," Computational and Mathematical Methods in Medicine, vol. 8, no. 3, pp. 153-164, 2007.

[30] Y. Y. Liu and Y. N. Xiao, "An epidemic model with saturated media/psychological impact," Applied Mathematics and Mechanics, vol. 34, no. 4, pp. 99-407, 2013.

[31] A. Wang and Y. Xiao, "A Filippov system describing media effects on the spread of infectious diseases," Nonlinear Analysis: Hybrid Systems, vol. 11, pp. 84-97, 2014.

[32] C. P. Bhunu, S. Mushayabasa, and H. Kojouharov, "Mathematical analysis of an HIV/AIDS model: impact of educational programs and abstinence in sub-Saharan Africa," Journal of
Mathematical Modelling and Algorithms, vol. 10, no. 1, pp. 31-55, 2011.

[33] Y. Wang, J. D. Cao, Z. Jin, H. F. Zhang, and G. Q. Sun, "Impact of media coverage on epidemic spreading in complex networks," Physica A: Statistical Mechanics and Its Applications, vol. 392, no. 23, pp. 5824-5835, 2013.

[34] X. Yuan, Y. Xue, and M. Liu, "Analysis of an epidemic model with awareness programs by media on complex networks," Chaos, Solitons \& Fractals, vol. 48, no. 1, pp. 1-11, 2013.

[35] S. Funk, E. Gilad, C. Watkins, and V. A. A. Jansen, "The spread of awareness and its impact on epidemic outbreaks," Proceedings of the National Academy of Sciences of the United States of America, vol. 106, no. 16, pp. 6872-6877, 2009.

[36] W. Liu, "A SIRS epidemic model incorporating media coverage with random perturbation," Abstract and Applied Analysis, vol. 2013, Article ID 792308, 9 pages, 2013.

[37] L. Wang, H. L. Huang, A. C. Xu, and W. M. Wang, "Stochastic extinction in an SIRS epidemic model incorporating media coverage," Abstract and Applied Analysis, vol. 2013, Article ID 891765, 8 pages, 2013.

[38] H. M. Wei, X. Z. Li, and M. Martcheva, "An epidemic model of a vector-borne disease with direct transmission and time delay," Journal of Mathematical Analysis and Applications, vol. 342, no. 2, pp. 895-908, 2008. 


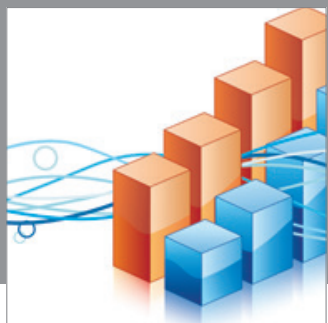

Advances in

Operations Research

mansans

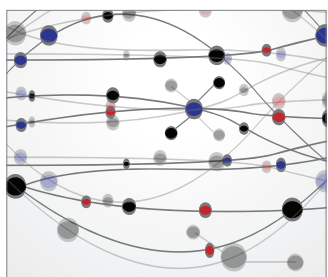

The Scientific World Journal
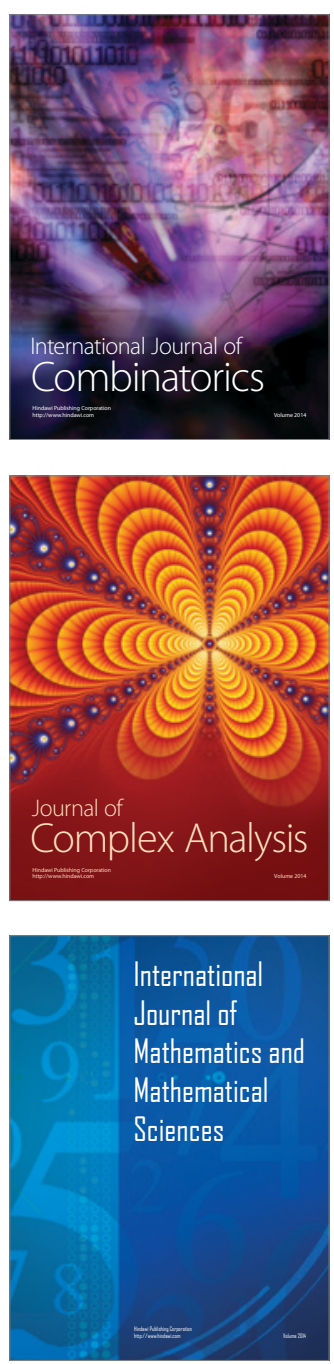
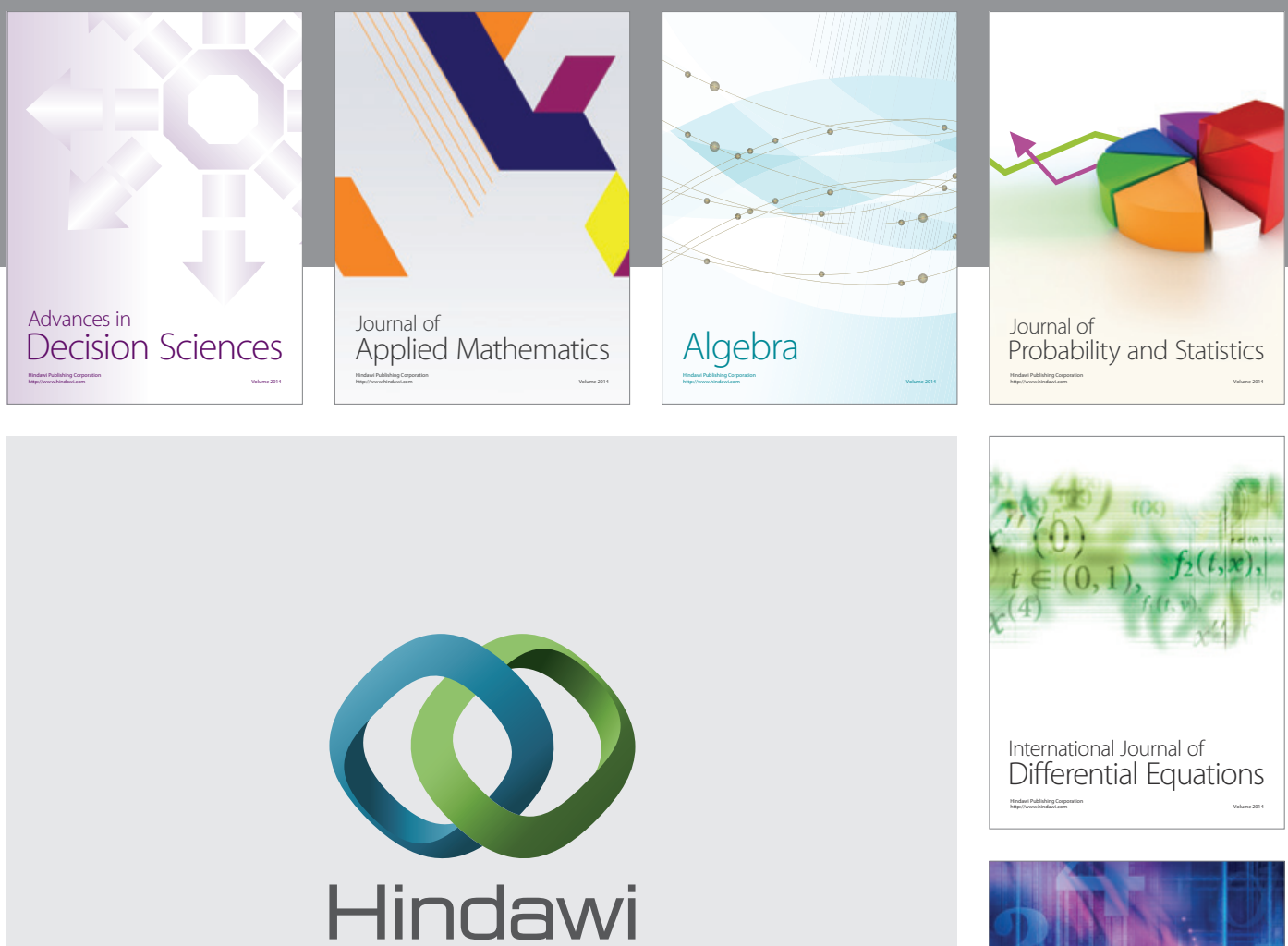

Submit your manuscripts at http://www.hindawi.com
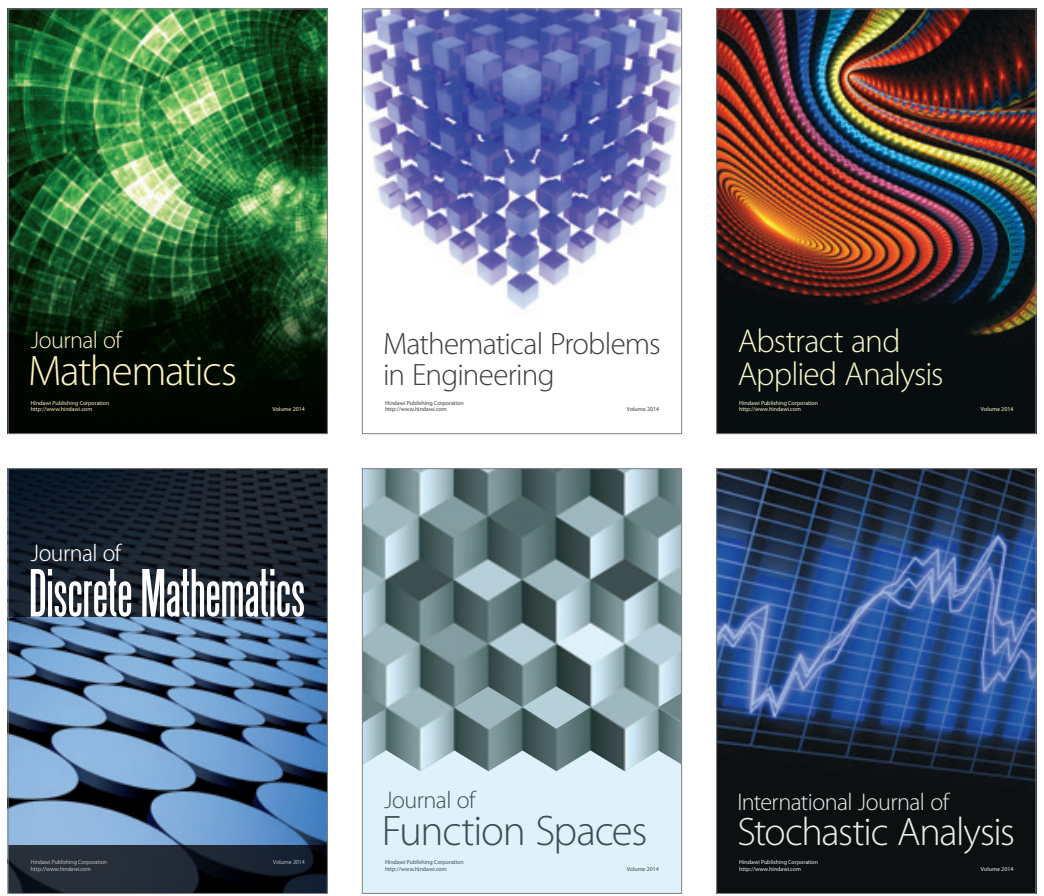

Journal of

Function Spaces

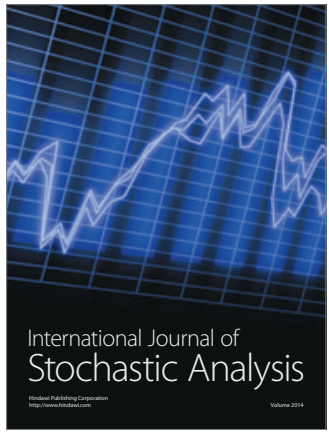

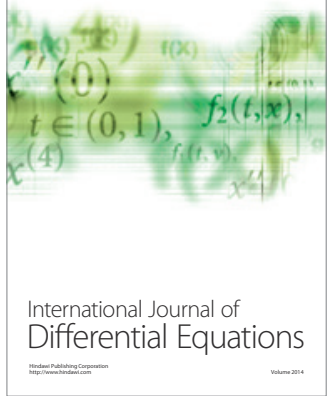
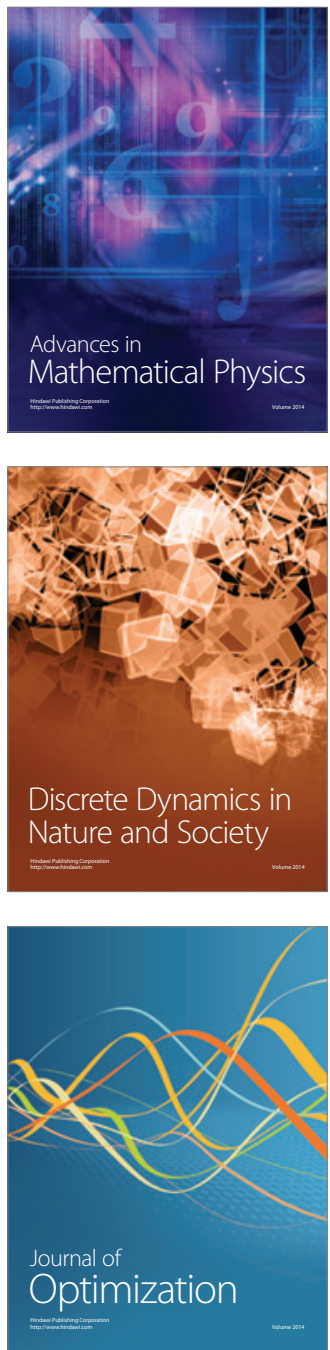